\title{
Concept of a 3D printed photopolymer casting models valuation algorithm in the aspect of Industry 4.0
}

\author{
Arkadiusz Kroma ${ }^{1}$, Robert Sika², Paweł Popielarski ${ }^{3}$ \\ \{arkadiusz.kroma@put.poznan.pl ${ }^{1}$, robert.sika@ put.poznan.pl ${ }^{1}$, pawel.popielarski@ put.poznan.pl ${ }^{1}$ \} \\ Poznan University of Technology, Piotrowo 3 street, 61-138 Poznan, Poland ${ }^{1}$
}

\begin{abstract}
The article presents an original concept of a 3D printed photopolymer model valuation algorithm - the APPMV algorithm (Algorithm of Photopolymer Printed Model Valuation). Both machine aspects and complexity of a model were taken into account, directly affecting the final time of prototype production. First part of the article briefly discusses the contemporary 3D printing technologies out of photopolymers, along with their application. The next part discusses the elements of the model's valuation algorithm in greater detail, with chronological concept of connections between these elements, in a form of a ready-to-use algorithm for the photopolymer 3D printing process. It turned out that the very important element of the presented algorithm is the Model Complexity Factor (MCF). Value of this factor depends on several elements described in the article, what makes it easier to estimate the final price of the offered product.
\end{abstract}

Keywords: 3D printing, photopolimers, prototype valuation, resin, Industry 4.0.

\section{Introduction}

Competitiveness of a modern enterprise is determined primarily by its ability to meet customer's needs in the best way possible. This means providing products or services, which meet client's requirements related to price, quality and, most recently, deadlines. Free market economy, where the role of the customer is dominant, makes these requirements more and more diversified and individualized, especially in complex production processes [1]. This is particularly evident in the era of Industry 4.0, which consists in developing special technologies accelerating human work, and personalizing orders for the target customers at the same time. This means testing and implementing new manufacturing processes based on automation, robotization and communication between machines without human intervention (on-line process) or with its larger / smaller share (off-line process) [2]. The effect of the above mentioned activities is a trend of Smart Factories design, where the entire product manufacturing process, from the prototype, through technology development, production planning, production control and service, will be digitally integrated (End-to-End process) [3]. 
The article [4] shows an overview of 3D printing technology currently used in foundry industry. However, due to relatively high accuracy and a large number of factors influencing the printing process, in this paper the authors focused only on the photopolymer printing technology. Therefore, the authors presented an original concept of an algorithm for valuation of a 3D printed photopolymer model - APPMV (Algorithm of Photopolymer Printed Model Valuation), that has to be used for valuation of 3D printed photopolymer models. Special attention has been paid to machine-specific aspects, such as the printing time, positioning or model complexity, which directly translates into the finishing time, known as the postprocessing. A very important element of the built algorithm was the Model Complexity Factor (MCF). The MCF value depends on several elements, described in the Chapter 4 of this paper. These listed elements make the final price of the offered product estimation much easier.

\section{Literature review}

The concept of Industry 4.0 assumes full human integration with the production machines on a digital level [5]. A Smart Factory should have a smart manufacturing environment, where machines communicate with each other in an automated way, passing key information to an operator. As a result, it will be possible to switch from the mass production to personalized production, accommodated to individual customer requirements [6] with maintaining low production costs - this is known as the Mass Customization [7].

Taking into account the above mentioned assumptions of Industry 4.0, the main focus should be put on the communication between the final user (a customer) and the business (a production company). This is very important in the current era of free market development, because the customers more and more often want to participate in the product manufacturing cycle, according to the business to customer principle (B2C) or the business to business principle (B2B), when the recipient is a production company, e.g. foundry [8]. In this situation, it is very important for a customer to obtain quick information about the price of the ordered products. The shorter the time from an inquiry submission to the offer, the greater chance to order fulfillment by a specific company.

The impact of selected production parameters on the final quality of the product is determined in foundries more and more. In the article [9], the authors propose the analysis of the ADI properties modeling to build a mathematical model predicting mechanical properties such as the tensile strength Rm [MPa] and elongation A5 [\%].Through application of the social machines, direct control of the process and supporting decisions at augmented operator's level to select quasi-optimal process parameters was possible.

Photopolymer 3D printing technology has existed for over 30 years, when Charles Hull (founder of one of the industry leaders - 3D Systems) has patented in 1986 (the patent process began in 1984) spatial models printing technology, based on irradiating with UV laser beam of an appropriate wavelength onto a surface of a liquid photopolymer, causing cross-linking reactions leading to solidification. This fundamental process is known as the stereolitography (SLA) [4].

In recent years, several new photopolymer 3D printing methods have been developed, such as the DPP (Daylight Polymer Printing), DLP (Digital Light Processing), DLS (Digital Light Synthesis), all based on the classic SLA process. The main differences are in technical details, such as the energy carrier or process dynamics. Among the methods described above, the oldest 
SLA technology is still the most commonly used photopolymer 3D printing process in the industry.

At least two variants of the patented SLA method can be distinguished - preferred and alternative solution (Fig. 1). Both versions involve the irradiation of a laser beam on a liquid photopolymer. The main difference is the XYZ coordinate system direction.

a)

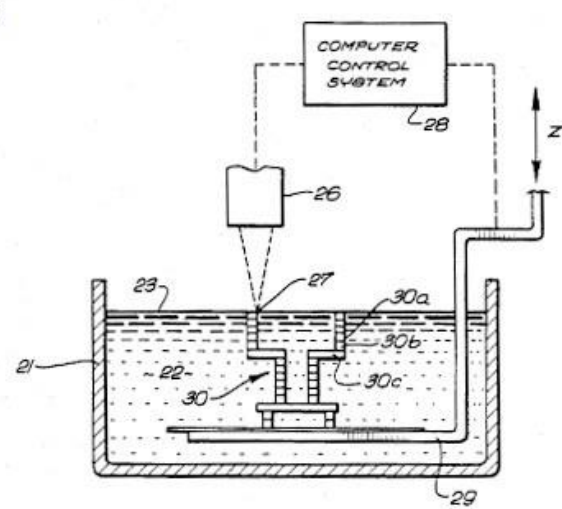

b)

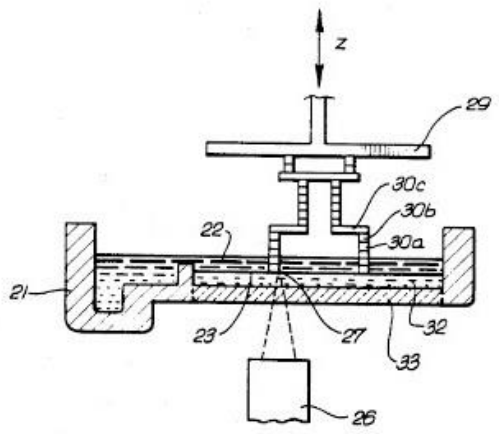

Fig. 1. SLA printer system solutions: a) preferred and b) alternative. Number labels used in the Figure: 21 - container, 22 - UV curable liquid, 23 - designated working surface, 26 - programmable source of ultraviolet light, 27 - spot of ultraviolet light, 28 - computer or other programming device, 29 - movable elevator platform, 30 - three-dimensional object, 30a/30b/30c - single layer, 32 - heavier UV transparent liquid, 33 - UV radiation passing through a suitable UV transparent window [10].

This technology is one of the most accurate (in terms of low roughness and dimensional repeatability) out of currently used 3D printing methods, and due to large number of resins with different properties such as increased hardness, flexibility, high temperature resistance or ash free burning off, has gained widespread use in industries such as:

- industrial design,

- jewelry / goldsmithing,

- mechanical engineering,

- medicine,

- casting.

In this article, the authors focused on the usage of SLA technology in the foundry industry. This method is used to prepare prototype models or pieces of products, out of a casting resin characterized by ash-free burning off. Due to possibility of fast, easy and relatively cheap (comparing to classic machining) printing of single 3D models, this process can be used as a substitute for the traditional method of lost wax casting, without having to create expensive matrices or patterns.

To make the photopolymer 3D printing process successful, it is the most important to fulfill the restrictive criteria related not only to the properly conducted design process of $3 \mathrm{D}$ models, but, above all, taking all the elements that have or may have a direct relationship with the printing process into account. Quantity, roles and mutual relations of individual elements of the photopolymer 3D printing process are presented in Chapter 3. 


\section{Research methodology}

Considering the aforementioned aspects regarding the SLA technology and the useage of photopolymer 3D printing in various industries, as well as complexity and number of elements having a direct impact on fast and properly conducted 3D printing process, the authors distinguished several key elements necessary for proper valuation of printing the casting models. The most important are:

- Construction correctness of the 3D model. In order to start the project valuation process, it is necessary to have a proper 3D model geometry, prepared, for instance, in the .STL format, containing triangular facet representation of surfaces in a three-dimensional space [11]. It should be mentioned that there are other acceptable formats of spatial geometry used in 3D printing processes, such as the .OBJ format. Regardless of the used format, the designed geometry should be continuous, uniform and with no surface discontinuities, otherwise an external interference in the file will be necessary to correct the errors.

- Total size of the final model. It is one of the basic elements affecting the printing time, directly related to the amount of resin used for production, including the volume of the model along with the support structures.

- Technological inclination angle of the model relative to the printing axis. The model inclination angle, also known as the orientation, determines the absolute height of the printed model (vertical hypotenuse projected onto the $\mathrm{Z}$ axis), which has a direct impact on the process time, as well as other characteristics of the obtained model [12]. This information also determines size and quantity of the required support structures.

- Layer thickness. Depending on the size of this parameter, the final product will have bigger or smaller side surface roughness, the printing process may also take longer or shorter time.

- Build volume and number of simultaneously printed models. Build volume of a machine determines the number of simultaneously printed models, it has also a direct impact on the amount of resin loss, resulting, e.g., from cleaning the tank, local intercalations (inclusions) of cured or detached resin etc.

- Size and quantity of supports. Support structures are necessary for a properly conducted 3D printing process. Depending on their shape, quantity and level of complexity, they affect, among others, the volume of the entire printed model, time of printing or the number of potential intercalations of the detached resin. In addition, they also affect the total time of the prototyping process, because it is necessary to remove them and clean the surface of the contact points.

- Density of supports. It affects the number of contact points and the finishing time.

- Machine operation time. This is the absolute working time of a 3D printer, without the post-processing and auxiliary production activities such as dismantling the platform with a ready printout or cleaning the resin tank, among others.

- Post-processing. Post-processing contains all the activities related to chemical-physical treatment of the already printed 3D model. The activities conducted during the post-processing include, among others: treatment with isopropyl alcohol (IPA) bath (depending on the used resin), exposure of the model to UV light for additional curing (this is known as the post-curing), removal of supports, sanding the support-model surface, etc.

- Overhead costs. These are the costs of used additional materials and tools, such as the sandpaper, balls, tongs, electricity, cost of the room, etc. 


\section{Results}

\subsection{Model Complexity Factor - MCF}

In this article, the authors assumed a simplified version of the Model Complexity Factor, which mathematically describes level of complexity of a model. Printing and further processing of difficult 3D models, i.e. having a large number of thin walls, a large number of irregular curves or a large number of openings, is associated with a great danger of model damage. Moreover, in the case of such non-technological designs, such solutions enforce intervention of an experienced operator at the production preparation stage, which is directly related to increase of the necessary model preparation time and the price of the final product.

Main elements of the MCF are: total support area at the point of contact with the initial model, ratio of the area of curves to straight surfaces, ratio of the printout volume to the initial volume of the model and more. When the value of MCF is smaller or similar to 1.0, model is easy to print, when the value is approx. 10, the model is very complicated. This aspect will be discussed in detail in another article.

\subsection{The APPMV algorithm}

The APPMV algorithm was developed on the basis of the assumptions presented in Chapter 3 of this paper. The authors also utilized their own experience in the field of preparing 3D printing processes, as well as in activities related to the printed model finishing. The individual steps of the algorithm are briefly described below.

STAGE 1: Automatic analysis of the model geometry for surface correctness. All the errors and surface discontinuities encountered by the program should be immediately identified, repaired (if possible) and the repaired model should be transferred back to the analysis (feedback).

STAGE 2: Construction of the final model geometry. The following elements should be considered: size and quantity of supports (SQS), density of supports (DS), technological inclination angle (TA). On the basis of values of these elements, the final volume of the printed model is calculated.

STAGE 3: Selection of the 3D printing machine, on the basis of the build volume, number of simultaneously printed models and layer thickness.

STAGE 4: Calculation of the machine operation time. This is the absolute working time of the 3D printer.

STAGE 5: Acceptance or modification of previous stages by the operator. At this stage, the human operator can make final corrections (if necessary) of the final model geometry or change any element from the previous stages, e.g. the inclination angle or the machine (3D printer).

STAGE 6: Printing of the model.

STAGE 7: Post-processing. The final stage of the algorithm includes manual work of the Augmented Operator, when the already printed model should be finished. It is necessary to consider additional work with the printed model, such as: IPA bath, removal of supports, sanding of the support contact points with the model, UV post-curing etc. The higher complexity of the model, the higher time of the finishing process and it can vary significantly, from minutes 
to hours. At this point, the authors propose to use the presented Model Complexity Factor $\mathrm{MCF}$, which helps the 3D printer owner to predict post-processing time and risk of printout damage.

The algorithm also includes overhead costs of the model production, such as: material price, tool wear (sandpaper, pliers, others) or cost of the room usage. The authors deliberately did not discuss the costs of various types of material, because the main assumption of this paper was printing of photopolymer casting models, which can be printed only from the special casting resin, without possibility of changing the material. The algorithm scheme is shown in the Fig. 3.

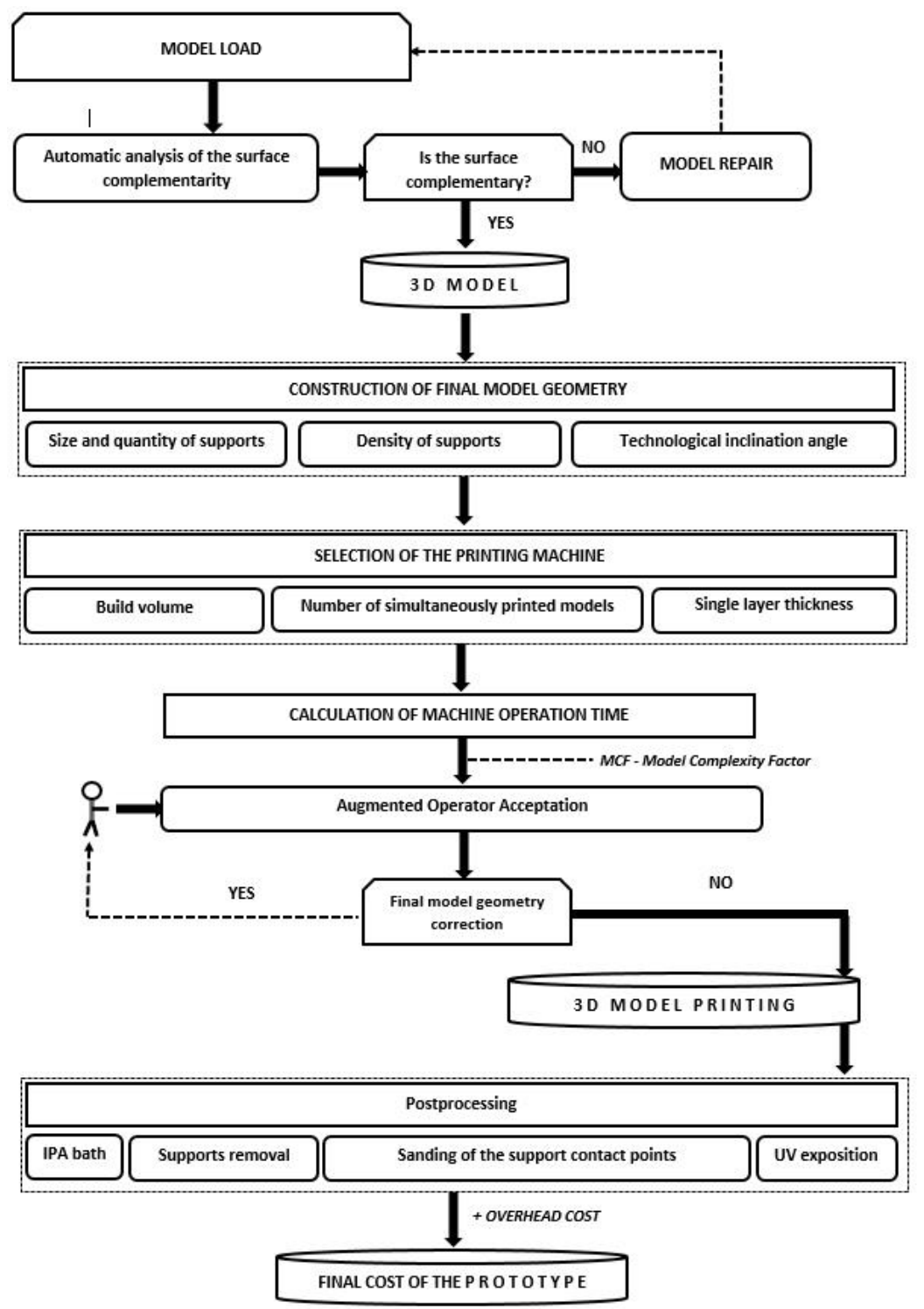

Fig. 2. Visualization of APPMV algorithm. 


\section{Conclusion}

In this article, the authors presented concept of the algorithm for valuation of $3 \mathrm{D}$ printed photopolymer casting models, which can be used as a basis for building an automatic valuation system. The concept of the algorithm should be treated as a guide to proper valuation of the photopolymer 3D printing, using as many elements as possible. Despite the high automation of processes in Industry 4.0, the authors paid particular attention on the important human factor in the form of the operator. Control over automated processes is still necessary, but the probability of errors generated automatically, despite their ever-smaller number, is still too high. The main role of the presented algorithm is to significantly shorten the time of the valuation process, that can have a direct impact on the growth of company's competitiveness.

\section{References}

[1] Ignaszak, Z., Sika, R., Rogalewicz, M.: Contribution to the Assessment of the Data Acquisition Effectiveness in the Aspect of Gas Porosity Defects Prediction in Ductile CastIron Castings. Vol. 18, Issue 1/2018, pp. 35-40. Achives of Foundry and Engineering (2018).

[2] Rojek, I., Kujawińska, A., Hamrol, A., Rogalewicz, M.: Artificial Neural Networks as a Means for Making Process Control Charts User Friendly, Vol. 637, pp. 168-178. International Conference on Intelligent Systems in Production Engineering and Maintenance, Springer (2017).

[3] Maddern, H., Smart, P.A.,Maukk, R.S., Childe, M\&S: End-to-end process management: Implications for theory and practice. Vol. 26, Issue 16, pp. 1303-1321. Production Planning \& Control, The Management of Operations (2013).

[4] Kroma A., Popielarski P., Szymański P.: Application of selected 3D printing methods in foundry industry. Advanced Manufacturing Technologies, Sozopol (2018).

[5] Gorecky, D., Schmitt, M., Loskyll M., Zuhlke D.: Human - machine - interaction in the Industry 4.0 ERA. Industrial Informatics (INDIN), 12th IEEE International Conference. pp. 289-294 (2014).

[6] Trojanowska J., Varela M.L.R., Machado J.: The Tool Supporting Decision Making Process in Area of Job-Shop Scheduling. In: Rocha Á., Correia A., Adeli H., Reis L., Costanzo S. (eds) Recent Advances in Information Systems and Technologies. WorldCIST. Advances in Intelligent Systems and Computing, vol. 571, pp. 490-498, Springer, Cham (2017).

[7] Górski F., Zawadzki P., Hamrol A.: Knowledge based engineering as a condition of effective mass production of configurable products by design automation, Journal of Machine Engineering, vol. 16, issue 4, pp. 5-30 (2016).

[8] Maruyam, H.: New trends in e-business: From B2B to Web Services. Vol. 20, Issue 1, pp. 125-139. New Generating Comuting, Computer Paradigms and Computational Intellingence, Springer (2002). 
[9] Kozłowski, J., Sika, R., Ciszak, O.: Modeling of Foundry Processes in the Era of Industry 4.0. Advances in Design, Simulation and Manufacturing. DSMIE. pp. 62-71 (2018).

[10] Hull, C.W.: Apparatus for production of three-dimensional objects by stereolithography. US4575330 patent US (1984).

[11] M.Szilvśi-Nagy, M., Mátyási, G.: Analysis of STL files. Vol. 38, Issues 7-9, pp. 945-960. Mathematical and Computer Modelling (2003).

[12] Górski, F., Wichniarek, R., Kuczko, W.: Strength of ABS parts produced by Fused Deposition Modelling technology - a critical orientation problem. Advances in Science and Technology-Research Journal Volume: 9 Issue: 26 pp: 12-19 (2015). 